\title{
Observation of the inverse spin Hall effect in the topological crystalline insulator SnTe using spin pumping
}

\author{
Shinobu Ohya, ${ }^{1,2,5, *}$ Akiyori Yamamoto, ${ }^{1}$ Tomonari Yamaguchi, ${ }^{3}$ Ryo Ishikawa, ${ }^{3}$ Ryota Akiyama, ${ }^{4}$ Le Duc Anh, $, 1,5$ \\ Shobhit Goel, ${ }^{1}$ Yuki K. Wakabayashi, ${ }^{1}$ Shinji Kuroda, ${ }^{3, \dagger}$ and Masaaki Tanaka ${ }^{1,2, \ddagger}$ \\ ${ }^{1}$ Department of Electrical Engineering and Information Systems, The University of Tokyo, \\ 7-3-1 Hongo, Bunkyo-ku, Tokyo 113-8656, Japan \\ ${ }^{2}$ Center for Spintronics Research Network, Graduate School of Engineering, The University of Tokyo, \\ 7-3-1 Hongo, Bunkyo-ku, Tokyo 113-8656, Japan \\ ${ }^{3}$ Institute of Materials Science, University of Tsukuba, 1-1-1 Tennoudai, Tsukuba 305-8573, Japan \\ ${ }^{4}$ Department of Physics, The University of Tokyo, 7-3-1 Hongo, Bunkyo-ku, Tokyo 113-0033, Japan \\ ${ }^{5}$ Institute of Engineering Innovation, Graduate School of Engineering, The University of Tokyo, \\ 7-3-1 Hongo, Bunkyo-ku, Tokyo 113-8656, Japan
}

(Received 6 May 2017; revised manuscript received 1 August 2017; published 20 September 2017)

\begin{abstract}
Topological crystalline insulator SnTe is a promising material for future spintronics applications because of the strong spin-orbit coupling and surface states protected by the mirror symmetry of the crystal. In this paper, using a high-quality epitaxial (001)-oriented $\mathrm{Fe} / \mathrm{SnTe} / \mathrm{CdTe} / \mathrm{ZnTe}$ heterostructure grown on GaAs, we successfully observe the inverse spin Hall effect in SnTe induced by spin pumping, which is confirmed by detailed analyses of the dependence of the electromotive force on the microwave power, magnetic-field angle, and temperature. By a rough estimation, a relatively large spin Hall angle of $\sim 0.01$ is obtained for bulk SnTe at room temperature. This large value may be partially caused by the surface states. Our result suggests that SnTe can be used for efficient spin-charge current conversion.
\end{abstract}

DOI: 10.1103/PhysRevB.96.094424

\section{INTRODUCTION}

Topological insulators (TIs) have attracted much attention due to their gapless metallic surface states (SSs) that are protected by time-reversal symmetry (TRS) [1-3]. The SSs are promising particularly because giant spin-charge current conversion efficiencies have been reported in TIs such as $\mathrm{Bi}_{2} \mathrm{Se}_{3}[4,5]$ and $\left(\mathrm{Bi}_{0.5} \mathrm{Sb}_{0.5}\right)_{2} \mathrm{Te}_{3}$ [6-8]. Meanwhile, because the SSs of TIs are protected by the TRS, they are easily broken when a ferromagnetic material is deposited on the surface of TIs due to a magnetic perturbation [9-12]. On the other hand, topological crystalline insulators (TCIs), which also have gapless metallic SSs, have received a lot of attention in recent years $[13,14]$. Unlike TIs, the SSs in TCIs are protected by the mirror reflection symmetry of the crystal. Thus, the influence of the breaking of TRS by magnetic perturbation is different in TCIs from that in other TIs. SnTe is a typical and promising TCI, in which the presence of the SSs has been experimentally confirmed by studies of angle-resolved photoemission spectroscopy, scanning tunnel spectroscopy, and electrical transports [15-25]. The strong spin-orbit coupling in bulk SnTe is also attractive for spintronic applications. However, there have been no reports of successful spin injection into either the SSs or the bulk state of SnTe.

One of the important requirements for the successful spin injection into SnTe is to obtain a high-quality ferromagnet/SnTe interface, which can suppress spin scattering at the interface during the spin injection from the ferromagnet. In this paper, we use an all-epitaxial single-crystalline Fe/SnTe mul-

\footnotetext{
*ohya@cryst.t.u-tokyo.ac.jp

†kuroda@ims.tsukuba.ac.jp

${ }^{\ddagger}$ masaaki@ee.t.u-tokyo.ac.jp
}

tilayer structure grown by molecular-beam epitaxy [Fig. 1(a)]. To demonstrate the spin-charge current conversion in SnTe, we use spin pumping, which is an efficient method to generate spin currents [26-28]. Under the ferromagnetic resonance (FMR) condition of the Fe layer, the induced magnetization precession generates a spin current, which is injected into SnTe, and holes are accumulated at a sample edge via the inverse spin Hall effect (ISHE) [Fig. 1(b)]. Note that SnTe exhibits a $p$-type metallic behavior [see Fig. 4(a)] and that the (spin) current is dominantly carried by holes in bulk SnTe, which is highly degenerated with high-density holes induced by $\mathrm{Sn}$ vacancies [22]. This charge accumulation is detected as an electromotive force (EMF) between the sample edges. However, we need a careful analysis of the EMF because galvanomagnetic effects such as planar Hall effect (PHE) and anomalous Hall effect (AHE), which are not directly related to the spin-charge current conversion, are incorporated in the EMF in addition to the ISHE [29,30]. Moreover, the EMF can be influenced by thermal effects such as Seebeck effect and longitudinal spinSeebeck effect (LSSE) [31,32]. Thus, in this paper, we carefully analyze the dependence of the EMF on the microwave power, magnetic-field angle, and temperature. We demonstrate a successful spin-charge current conversion in SnTe.

\section{SAMPLE AND EXPERIMENTAL SETUP}

The structure used in our paper is composed of $\mathrm{Fe}$ $(20 \mathrm{~nm}) / \mathrm{SnTe}(70 \mathrm{~nm}) / \mathrm{CdTe}(550 \mathrm{~nm}) / \mathrm{ZnTe}(2 \mathrm{~nm})$ grown on a GaAs (001) substrate without exposure to the air during the growth [Fig. 1(a)] [33]. The reflection high-energy electrondiffraction patterns of the SnTe and Fe layers and the x-raydiffraction spectrum show that these layers are epitaxially grown on $\mathrm{CdTe} / \mathrm{ZnTe} / \mathrm{GaAs}$ [Figs. 1(c)-1(e)]. From the Hall measurement on another SnTe film (without an $\mathrm{Fe}$ layer) 

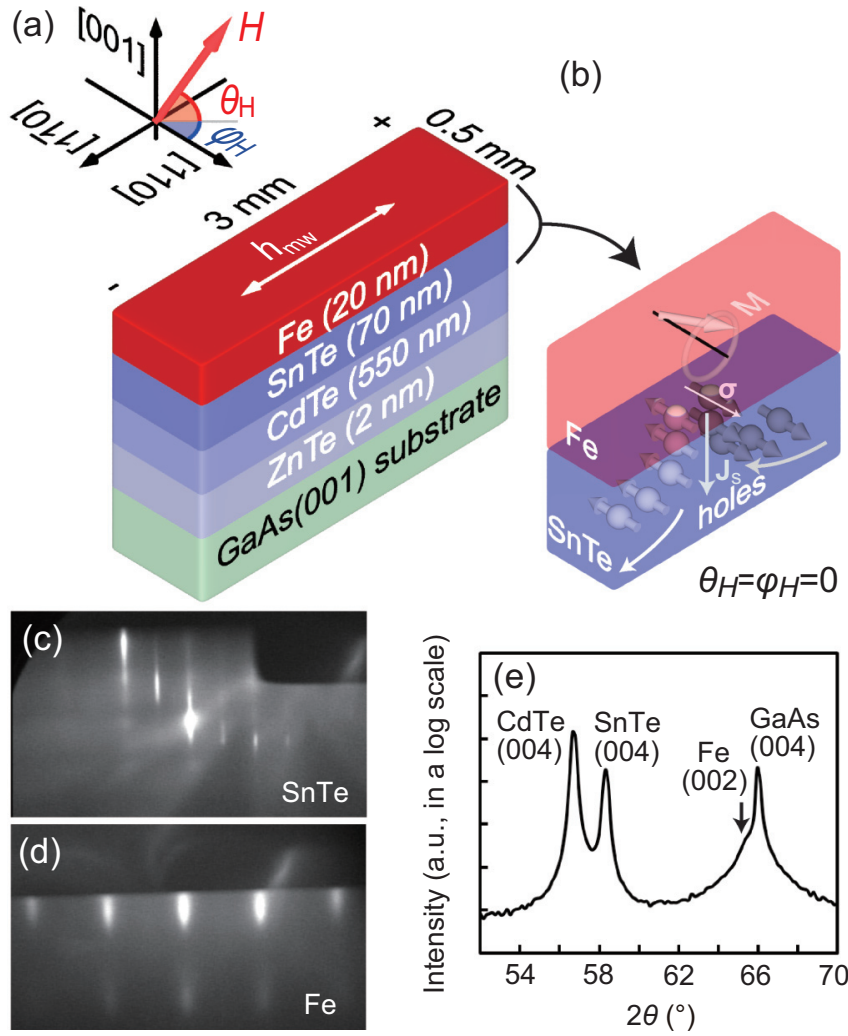

FIG. 1. (a) Schematic illustration of the (001)-oriented fullepitaxial multilayer structure composed of $\mathrm{Fe} / \mathrm{SnTe} / \mathrm{CdTe} / \mathrm{ZnTe}$ grown on $\operatorname{GaAs}(001)$ substrate. The sample was cut into small pieces with a size of $3 \times 0.5 \mathrm{~mm}$. In the electron-spin-resonance system, a rf magnetic field $h_{\mathrm{mw}}$ was applied along the [110] direction of the sample. The out-of-plane angle of the magnetic field $H$ is defined as $\theta_{H}$ with respect to the film plane. The in-plane angle of $H$ is defined as $\varphi_{H}$ with respect to the [110] direction in the film plane. (b) Mechanism of the generation of the spin current and the EMF. The vectors $\mathbf{J}_{\mathrm{s}}$ and $\boldsymbol{\sigma}$ represent the spin current and spin polarization of the spin current, respectively. Under the FMR condition of the Fe layer, the induced precession of the magnetization (M), which is represented by the thick white arrow in the Fe layer, generates a spin current. This is injected into SnTe and accumulated at a sample edge by the ISHE. (c,d) Reflection high-energy electron-diffraction patterns observed after the growth of the SnTe layer (c) and the Fe layer (d), when the electron-beam azimuth is the [110] direction. (e) X-ray-diffraction spectrum $(\theta-2 \theta$ scan) for the Fe/SnTe sample.

grown in the same condition, the density and the mobility of bulk holes are estimated approximately at $5 \times 10^{20} \mathrm{~cm}^{-3}$ and $100 \mathrm{~cm}^{2} / \mathrm{V} \mathrm{s}$ at $4 \mathrm{~K}$, respectively. This result suggests that the contribution of the bulk holes to the electrical transport is dominant over that of the two-dimensional carriers of the SSs. We have carried out spin pumping measurements using a $\mathrm{TE}_{011}$ cavity of an electron-spin-resonance system (microwave frequency: $9.1 \mathrm{GHz}$ ). We cut the sample into small pieces with a size of $3 \times 0.5 \mathrm{~mm}$ and put a sample piece at the center of the cavity. The EMF is defined as the voltage of the edge of the Fe layer in the [110] direction of the GaAs substrate with respect to that of the opposite side (i.e., in the [1ㅣㅣㄹ direction) as shown in Fig. 1(a). The used microwave power was $200 \mathrm{~mW}$
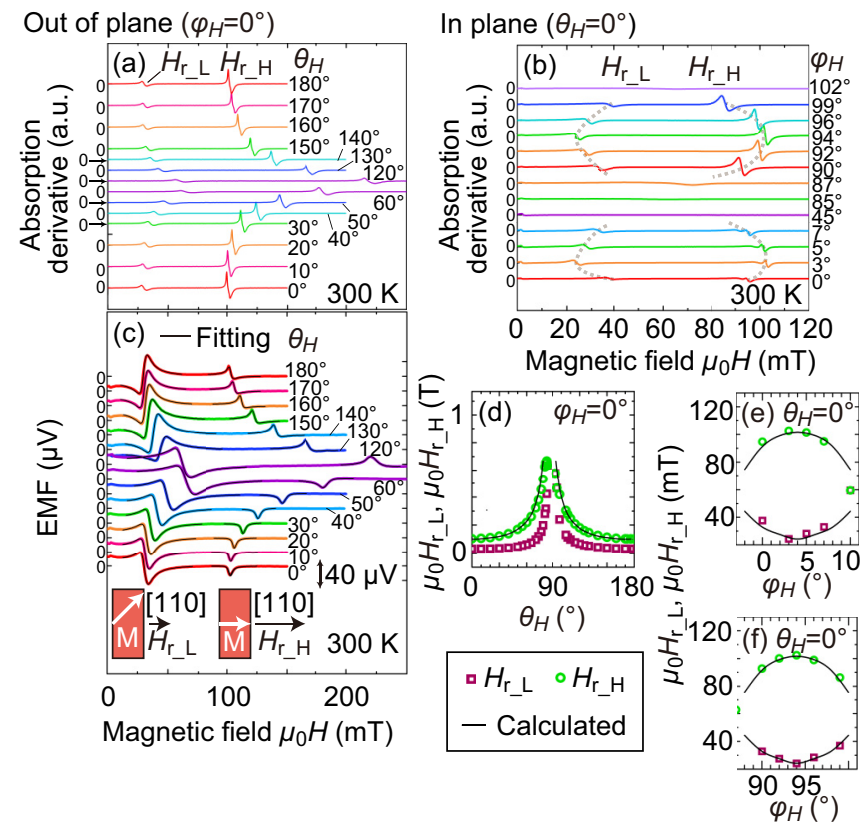

FIG. 2. (a,b) Magnetic-field $\mu_{0} H$ dependence of the microwave absorption derivative for various $\theta_{H}$ when $\varphi_{H}=0^{\circ}$ (a) and for various $\varphi_{H}$ when $\theta_{H}=0^{\circ}$ (b). In (b), the gray dotted curves represent the traces of the resonance magnetic fields. (c) $\mu_{0} H$ dependence of the EMF for various $\theta_{H}$ when $\varphi_{H}=0^{\circ}$. The black curves are fitting curves expressed by the sum of the symmetric and antisymmetric curves. (d) Higher and lower resonance magnetic fields $\left(H_{\mathrm{r}_{-} \mathrm{H}}, H_{\mathrm{r}_{-} \mathrm{L}}\right)$ as a function of $\theta_{H}$ when $\varphi_{H}=0^{\circ}$. (e,f) $H_{\mathrm{r}_{-} \mathrm{H}}$ and $H_{\mathrm{r}_{-} \mathrm{L}}$ as a function of $\varphi_{H}$ around $\varphi_{H}=0^{\circ}$ (e) and $\varphi_{H}=90^{\circ}$ (f) when $\theta_{H}=0^{\circ}$. In (d), (e), and (f), the black curves express the calculated resonance magnetic fields. All the data shown in Fig. 1 are measured at $300 \mathrm{~K}$. The used power of the microwave was $200 \mathrm{~mW}$.

except for the measurements of the power dependence of the EMF shown in Fig. 4(d).

\section{RESULTS AND DISCUSSION}

In the single-crystal Fe layer, the magnetization direction under the FMR condition is strongly influenced by the magnetocrystalline anisotropy, which also influences the EMF signal as explained later. To understand this effect, we measured the FMR spectrum of our sample applying a magnetic field $H$ in various directions. As shown in Fig. 1(a), the out-of-plane angle of $H$ is defined as $\theta_{H}$ with respect to the film plane, and the in-plane angle is defined as $\varphi_{H}$ with respect to the [110] direction in the film plane of the GaAs substrate. As shown in Figs. 2(a) and 2(b), most of the measured FMR spectra show two resonances at magnetic fields $H_{\mathrm{r}_{-} \mathrm{L}}$ and $H_{\mathrm{r}_{-} \mathrm{H}}$ $\left(H_{\mathrm{r}_{-} \mathrm{L}}<H_{\mathrm{r}_{-} \mathrm{H}}\right)$. When $H$ is rotated out of plane at $\varphi_{H}=0^{\circ}$ as shown in Fig. 2(a), $H_{\mathrm{r}_{-} \mathrm{L}}$ and $H_{\mathrm{r}_{-} \mathrm{H}}$ tend to be extremely large near $\theta_{H}=90^{\circ}$, which reflects the strong in-plane magnetic anisotropy of the Fe film. When $H$ is rotated in the film plane as shown in Fig. 2(b), the resonance magnetic fields change with a period of $90^{\circ}$ (see the gray dotted curves, which trace the resonance magnetic fields). This is caused by the fourfold magnetocrystalline anisotropy with the easy axes along the in-plane $\langle 100\rangle$ directions in the Fe layer [34,35]. 

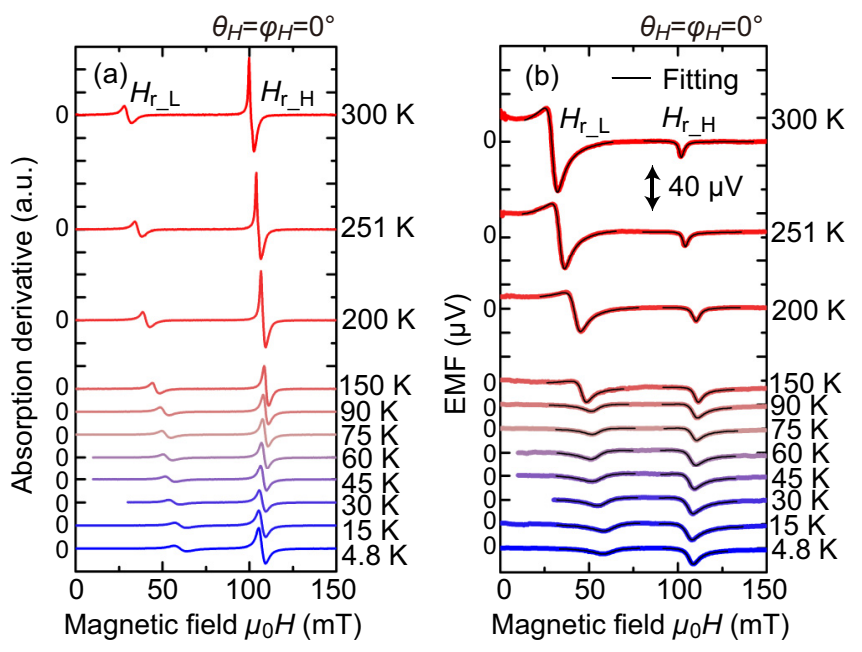

FIG. 3. (a,b) $\mu_{0} H$ dependences of the microwave absorption derivative (a) and the EMF (b) at various temperatures when $\theta_{H}=$ $\varphi_{H}=0^{\circ}$. The used power of the microwave was $200 \mathrm{~mW}$. In (b), the black curves are fitting curves expressed by the sum of the symmetric and antisymmetric curves.

(Both $\varphi_{H}$ and $\theta_{H}$ have a small deviation of $\sim 4^{\circ}$ from the crystallographic orientation due to a small misalignment of the sample.) As a result, as shown in the insets of Fig. 2(c), when $\theta_{H}=\varphi_{H}=0^{\circ}$ [see the bottom red curve in Fig. 2(a)], the magnetization direction is different from the direction of $H$ (i.e., [110] of the GaAs substrate) and is along one of the $\langle 100\rangle$ directions at $H=H_{\mathrm{r}_{-} \mathrm{L}}$, while the magnetization is aligned along the $H$ direction at $H=H_{\mathrm{r}_{-} \mathrm{H}}$. In fact, the $\theta_{H}$ and $\varphi_{H}$ dependences of $H_{\mathrm{r}_{-} \mathrm{L}}$ and $H_{\mathrm{r}_{-} \mathrm{H}}$ are well reproduced using the typical cubic and uniaxial out-of-plane anisotropy constants of the single-crystal Fe layer $(27.5 \mathrm{mT}$ and $-130 \mathrm{mT}$, respectively) as shown by the black curves in Figs. 2(d)-2(f) [34]. [The reason for the difference of the FMR spectra at $\theta_{H}=\varphi_{H}=0^{\circ}$ between Figs. 2(a) and 2(b) may be due to the different sample alignments used for these experiments. For more details of the calculation of the resonance fields and the shapes of the FMR spectra, see Secs. I and II in the Supplemental Material, respectively [36]].

The EMF- $H$ curves also exhibit two peaks corresponding to the resonances observed in the FMR spectra [Fig. 2(c)], which indicates that the EMF is induced by FMR. The shapes of the EMF- $H$ curves are almost symmetric between $\theta_{H}=0$ and $180^{\circ}$. This result is consistent with the ISHE that is expressed by $\mathbf{J}_{\mathrm{s}} \times \boldsymbol{\sigma},[37,38]$ where $\mathbf{J}_{\mathrm{s}}$ and $\boldsymbol{\sigma}$ are the spin current and spin-polarization vector of the spin current [Fig. 1(a)]. The negative sign of the EMF peaks when $\theta_{H}=0^{\circ}$ [see the bottom red curve in Fig. 2(c)] is thought to be associated with the p-type conduction of bulk SnTe [28,39].

By analyzing the temperature dependence of the FMR- $H$ and EMF- $H$ curves of our Fe/SnTe sample obtained at $\theta_{H}=$ $\varphi_{H}=0^{\circ}$ shown in Figs. 3(a) and 3(b), we can qualitatively understand the origins of the EMF signals. Comparing Fig. 3(a) with Fig. 3(b), we see that the EMF peaks are observed at resonance magnetic fields at all the temperatures, which again indicates that the EMF is induced by FMR. While the EMF peak intensity at $H_{\mathrm{r}_{-} \mathrm{L}}$ strongly depends on the
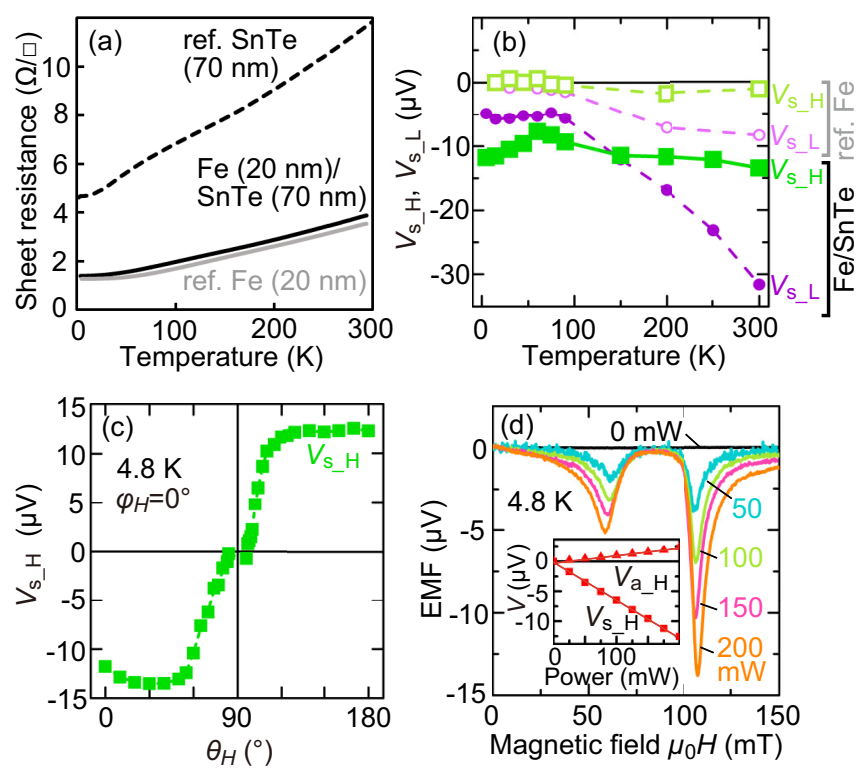

FIG. 4. (a) Sheet resistances of the Fe $(20 \mathrm{~nm}) / \mathrm{SnTe}(70 \mathrm{~nm}) /$ $\mathrm{CdTe} / \mathrm{ZnTe} / \mathrm{GaAs}$ sample (black solid curve), reference Fe $(20 \mathrm{~nm}) /$ GaAs sample (gray curve), and reference SnTe $(70 \mathrm{~nm}) / \mathrm{CdTe} /$ $\mathrm{ZnTe} / \mathrm{GaAs}$ sample (black broken curve). (b) Temperature dependences of $V_{\mathrm{s}_{-} \mathrm{L}}$ and $V_{\mathrm{s}_{-} \mathrm{H}}$ for the $\mathrm{Fe}(20 \mathrm{~nm}) / \mathrm{SnTe}(70 \mathrm{~nm}) /$ $\mathrm{CdTe} / \mathrm{ZnTe} / \mathrm{GaAs}$ sample and for the reference Fe $(20 \mathrm{~nm}) / \mathrm{GaAs}$ sample. (c) Out-of-plane magnetic-field angle $\theta_{\mathrm{H}}$ dependence of $V_{\mathrm{s}_{-} \mathrm{H}}$ at $4.8 \mathrm{~K}$. Here, $\varphi_{\mathrm{H}}$ was fixed at $0^{\circ}$. In (b) and (c), the used microwave power was $200 \mathrm{~mW}$. (d) $\mu_{0} H$ dependence of the EMF for various microwave powers at $4.8 \mathrm{~K}$. The inset shows the derived symmetric and antisymmetric components $\left(V_{\mathrm{s}_{-} \mathrm{H}}, V_{\mathrm{a}_{-} \mathrm{H}}\right)$ of the EMF at the higher resonance magnetic field of $107.6 \mathrm{mT}$ as a function of the microwave power at $4.8 \mathrm{~K}$.

temperature, the one at $H_{\mathrm{r}_{-} \mathrm{H}}$ does not. As explained below, the strong temperature dependence at $H_{\mathrm{r}_{-} \mathrm{L}}$ is attributed to the $\mathrm{PHE}$, which is proportional to $\overline{\operatorname{Re}\left[(\mathbf{M} \cdot \boldsymbol{\varepsilon}) M_{[\overline{1} 10]}\right]}$. Here, the overbar denotes time averaging, and $\mathbf{M}$ is the sum of the static magnetization and rf magnetization, which is much smaller than the static magnetization because the rf magnetic field $h_{\mathrm{mw}}$ is very small $(0.16 \mathrm{mT}) . \varepsilon$ is the rf electric field along the [110] direction, which should be zero at the center of the cavity but exists when the sample position is deviated from the center. The absolute value of $\boldsymbol{\varepsilon}$ depends on the deviation of the sample position from the center. $M_{[110]}$ is the [110] direction component of $\mathbf{M}$. When $\mathbf{M}$ is along the [110] direction (i.e., at $\left.H=H_{\mathrm{r}_{-} \mathrm{H}}\right), M_{[\overline{1} 10]}$ is composed of only the small rf magnetization, and thus the influence of the PHE is small. However, when $\mathbf{M}$ is not along the [110] direction like the case of $H=H_{\mathrm{r}_{-} \mathrm{L}}, M_{[\overline{1} 10]}$ has the [110] direction component of the static magnetization, and the PHE largely affects the EMF signal. It is known that the PHE and AHE are proportional to $R^{n}$. Here, $R$ is the resistance of the Fe layer and $n$ is $1-2$, which is determined by the scattering mechanism. Thus, due to the decrease in $R$ with decreasing temperature [see the gray solid curve in Fig. 4(a) measured for a reference single-crystal Fe layer directly grown on a semi-insulating (SI) GaAs (001) substrate], the AHE and PHE decrease with decreasing temperature [40]. Therefore, the strong temperature dependence of the EMF peak intensity at 
$H=H_{r_{-} \mathrm{L}}$ can be attributed to these galvanomagnetic effects. On the other hand, we see nearly a constant EMF at $H=H_{\mathrm{r}_{-} \mathrm{H}}$ in the entire temperature region despite the large change of $R$. This is clearly different from the behavior of the PHE and AHE of Fe, which indicates that the EMF signal at $H_{\mathrm{r}_{-} \mathrm{H}}$ is not affected by these galvanomagnetic effects.

For understanding the above-mentioned scenario quantitatively, it is useful to separate the $V-H$ curves into a symmetric (Lorentzian) component $V_{\mathrm{s}_{-} i}$ and an antisymmetric (diffusive) component $V_{\mathrm{a}_{-} i}$ at the resonance magnetic fields $H=H_{\mathrm{r}_{-} i}$ ( $i=\mathrm{H}, \mathrm{L}$ ) [30]. [In Figs. 2(c) and 3(b), the thin black curves are the fitting curves expressed by the sum of the symmetric and antisymmetric curves]. It is known that PHE, AHE, and ISHE are incorporated in $V_{\mathrm{s}_{-} i}$, while $V_{\mathrm{a}_{-} i}$ is composed only of PHE and AHE. In Fig. 4(b), as mentioned above, while $V_{\mathrm{s}_{-} \mathrm{L}}$ shows a strong temperature dependence due to the galvanomagnetic effects, $V_{\mathrm{s}_{-} \mathrm{H}}$ is almost constant as a function of the temperature, which is different from the behavior of PHE and AHE. Furthermore, to confirm our understanding, we have carried out the same spin pumping experiments on the reference single-crystal Fe (20 nm)/SI GaAs sample [Fig. 4(b)]. Because $\mathrm{GaAs}$ is insulating, no spin current is injected into GaAs in this reference sample, and we can estimate the influences of the $\mathrm{PHE}$ and $\mathrm{AHE}$ in the Fe layer. Indeed, in this reference sample, while $V_{\mathrm{s}_{-} \mathrm{L}}$ shows a strong temperature dependence due to the PHE (or AHE), $V_{\mathrm{s}_{-} \mathrm{H}}$ is almost zero and does not depend on the temperature. Although the absolute values of the PHE and AHE components are different between the two samples possibly due to a small deviation of the sample position, their temperature dependences should be the same. Therefore, in the $\mathrm{Fe} / \mathrm{SnTe}$ sample, the PHE and AHE components are negligibly small in $V_{\mathrm{s}_{-} \mathrm{H}}$. (See also the discussions about the behavior of $V_{\mathrm{s}_{-} \mathrm{L}}, V_{\mathrm{a}_{-} \mathrm{L}}$, and $V_{\mathrm{a}_{-} \mathrm{H}}$ in Sec. III in the Supplemental Material [36]).

As discussed above, the contribution of the PHE and AHE components can be excluded in $V_{\mathrm{s}_{-} \mathrm{H}}$ from its temperature dependence. In addition, we can also exclude the influence of thermal effects on the EMF as shown below. As shown in Fig. 4(c), one can see that the $\theta_{H}$ dependence of $V_{\mathrm{s}_{-} \mathrm{H}}$ shows a typical behavior of the ISHE expressed by $\mathbf{J}_{s} \times \boldsymbol{\sigma}$; the sign of $V_{\text {s_H }}$ is reversed with the reversal of the in-plane component of the magnetic field (or $\sigma$ ). From this result, we see that the Seebeck effect, which is not related to the magnetization direction, is negligibly small in $V_{\mathrm{S}_{-} \mathrm{H}}$. This is also evidenced by the microwave-power dependence of the EMF shown in Fig. 4(d), in which EMF $\left(V_{\mathrm{a}_{-} \mathrm{H}}\right.$ and $\left.V_{\mathrm{s}_{-} \mathrm{H}}\right)$ is proportional to the microwave power [see the inset of Fig. 4(d)]. The EMF signal induced by the Seebeck effect would be proportional to $S \Delta T$ [41], where $S$ is the Seebeck coefficient of $S n T e$ and $\Delta T$ is the temperature difference induced in the sample by microwave irradiation. Because $S$ strongly depends on the temperature in SnTe [42], the power dependence of the EMF would not be linear if it were affected by the Seebeck effect. We can also eliminate the influence of the LSSE, which is caused by the spin current that is induced by the temperature difference between the top and bottom interfaces of the Fe layer $[31,32]$, because the specific heat of Fe strongly decreases to zero with decreasing temperature, which is different from the nearly constant temperature dependence of $V_{\mathrm{s}_{-} \mathrm{H}}$ [Fig. 4(b)]. Therefore, we can conclude that $V_{\mathrm{s}_{-} \mathrm{H}}$ originates from the ISHE over the entire temperature range used in our paper.

Finally, we roughly estimate the spin Hall angle of SnTe. The FMR spectral width of the absorption derivative at $H=$ $H_{r_{-} \mathrm{H}}$ is $4.35 \mathrm{mT}$ when $\theta_{H}=\varphi_{H}=0^{\circ}$ at room temperature [see Fig. 2(a)]. In our reference single-crystal Fe/GaAs sample, the obtained spectrum width of the absorption derivative was $4.13 \mathrm{mT}$. From these values, the mixing conductance is estimated to be $1.3 \times 10^{19} \mathrm{~m}^{-2}$, and the Gilbert damping constant is estimated to be 0.013 . If we assume that the spin-diffusion length of SnTe is the same as that of $\mathrm{Bi}_{2} \mathrm{Se}_{3}(\sim 6 \mathrm{~nm})$ [44], the spin Hall angle is roughly estimated to be $\sim 0.013$ for $\mathrm{SnTe}$ [43]. This is much larger than those of $\mathrm{Si}$ and $\mathrm{GaAs}$, and is the same order of magnitude as those of the bulk $\mathrm{Bi}_{2} \mathrm{Se}_{3}$ [44] and heavy metal Pd [43]. Thus, the bulk state of SnTe is promising for future spintronics applications. This large value may be partially caused by the presence of the SS. In our experiments, it is difficult to distinguish the contribution of the SSs to the EMF signal due to the large conductivity of the bulk state of $\mathrm{SnTe}$. For the detection of the signal originating from the SSs, systematic measurements of the dependence of the EMF on the SnTe layer thickness are required; however, in the present growth technique, we need a thick SnTe layer to obtain the high-quality crystal and interface of $\mathrm{Fe} / \mathrm{SnTe}$, which are important to achieve efficient spin injection into SnTe from Fe. Further improvement of the growth conditions to reduce the bulk conduction is required for exploiting the full potential of $\mathrm{SnTe}$.

\section{SUMMARY}

In summary, we have carried out spin pumping experiments on the all-epitaxial Fe/SnTe multilayer structure. We have successfully injected a spin current into SnTe from Fe using spin pumping under the FMR condition of the Fe layer. By careful and detailed analyses of the dependence of the EMF on the microwave power, magnetic-field angle, and temperature, we have concluded that the EMF peak obtained at $H_{r_{-} H}$ is induced by the ISHE in bulk SnTe. A relatively large spin Hall angle of $\sim 0.01$ is estimated for bulk SnTe. This large value may be partially caused by the SSs. Our result indicates that SnTe is very promising for future spintronic applications.

\section{ACKNOWLEDGMENTS}

This paper was supported by Grants-in-Aid for Scientific Research (Grants No. 26103003, No. 26249039, and No. 16H00983); Cooperative Research Project Program of RIEC; Tohoku University; Project for Developing Innovation Systems of MEXT; and Spintronics Research Network of Japan.
[1] M. Z. Hasan and C. L. Kane, Rev. Mod. Phys. 82, 3045 (2010).
[2] X.-L. Qi and S.-C. Zhang, Rev. Mod. Phys. 83, 1057 (2011). 
[3] Y. Ando, J. Phys. Soc. Jpn. 82, 102001 (2013).

[4] A. R. Mellnik, J. S. Lee, A. Richardella, J. L. Grab, P. J. Mintun, M. H. Fischer, A. Vaezi, A. Manchon, E.-A. Kim, N. Samarth, and D. C. Ralph, Nature (London) 511, 449 (2014).

[5] L. Liu, A. Richardella, I. Garate, Y. Zhu, N. Samarth, and C.-T. Chen, Phys. Rev. B 91, 235437 (2015).

[6] Y. Fan, P. Upadhyaya, X. Kou, M. Lang, S. Takei, Z. Wang, J. Tang, L. He, L.-T. Chang, M. Montazeri, G. Yu, W. Jiang, T. Nie, R. N. Schwartz, Y. Tserkovnyak, and K. L. Wang, Nat. Mater. 13, 699 (2014).

[7] K. Kondou, R. Yoshimi, A. Tsukazaki, Y. Fukuma, J. Matsuno, K. S. Takahashi, M. Kawasaki, Y. Tokura, and Y. Otani, Nat. Phys. 12, 1027 (2016).

[8] H. Wang, J. Kally, J. S. Lee, T. Liu, H. Chang, D. R. Hickey, K. A. Mkhoyan, M. Wu, A. Richardella, and N. Samarth, Phys. Rev. Lett. 117, 076601 (2016).

[9] L. A. Wray, S.-Y. Xu, Y. Xia, D. Hsieh, A. V. Fedorov, Y. S. Hor, R. J. Cava, A. Bansil, H. Lin, and M. Z. Hasan, Nat. Phys. 7, 32 (2011)

[10] S.-Y. Xu, M. Neupane, C. Liu, D. Zhang, A. Richardella, L. A. Wray, N. Alidoust, M. Leandersson, T. Balasubramanian, J. Sánchez-Barriga, O. Rader, G. Landolt, B. Slomski, J. H. Dil, J. Osterwalder, T.-R. Chang, H.-T. Jeng, H. Lin, A. Bansil, N. Samarth, and M. Z. Hasan, Nat. Phys. 8, 616 (2012).

[11] C.-Z. Chang, J. Zhang, X. Feng, J. Shen, Z. Zhang, M. Guo, K. Li, Y. Ou, P. Wei, L.-L. Wang, Z.-Q. Ji, Y. Feng, S. Ji, X. Chen, J. Jia, X. Dai, Z. Fang, S.-C. Zhang, K. He, Y. Wang, L. Lu, X.-C. Ma, and Q.-K. Xue, Science 340, 167 (2013).

[12] H. T. He, G. Wang, T. Zhang, I. K. Sou, G. K. L. Wong, J. N. Wang, H. Z. Lu, S. Q. Shen, and F. C. Zhang, Phys. Rev. Lett. 106, 166805 (2011).

[13] L. Fu, Phys. Rev. Lett. 106, 106802 (2011).

[14] T. H. Hsieh, H. Lin, J. Liu, W. Duan, A. Bansil, and L. Fu, Nat. Commun. 3, 982 (2012).

[15] P. Dziawa, B. J. Kowalski, K. Dybko, R. Buczko, A. Szczerbakow, M. Szot, E. Łusakowska, T. Balasubramanian, B. M. Wojek, M. H. Berntsen, O. Tjernberg, and T. Story, Nat. Mater. 11, 1023 (2012).

[16] Y. Tanaka, Z. Ren, T. Sato, K. Nakayama, S. Souma, T. Takahashi, K. Segawa, and Y. Ando, Nat. Phys. 8, 800 (2012).

[17] I. Zeljkovic, Y. Okada, C.-Y. Huang, R. Sankar, D. Walkup, W. Zhou, M. Serbyn, F. Chou, W.-F. Tsai, H. Lin, A. Bansil, L. Fu, M. Z. Hasan, and V. Madhavan, Nat. Phys. 10, 572 (2014).

[18] C. Yan, J. Liu, Y. Zang, J. Wang, Z. Wang, P. Wang, Z.-D. Zhang, L. Wang, X. Ma, S. Ji, K. He, L. Fu, W. Duan, Q.-K. Xue, and X. Chen, Phys. Rev. Lett. 112, 186801 (2014).

[19] Y. Tanaka, T. Shoman, K. Nakayama, S. Souma, T. Sato, T. Takahashi, M. Novak, K. Segawa, and Y. Ando, Phys. Rev. B 88, 235126 (2013).

[20] Y. Tanaka, T. Sato, K. Nakayama, S. Souma, T. Takahashi, Z. Ren, M. Novak, K. Segawa, and Y. Ando, Phys. Rev. B 87, 155105 (2013).

[21] I. Zeljkovic, D. Walkup, B. A. Assaf, K. L. Scipioni, R. Sankar, F. Chou, and V. Madhavan, Nat. Nanotech. 10, 849 (2015).
[22] R. Akiyama, K. Fujisawa, T. Yamaguchi, R. Ishikawa, and S. Kuroda, Nano Res. 9, 490 (2016).

[23] B. A. Assaf, F. Katmis, P. Wei, B. Satpati, Z. Zhang, S. P. Bennett, V. G. Harris, J. S. Moodera, and D. Heiman, Appl. Phys. Lett. 105, 102108 (2014).

[24] M. Safdar, Q. Wang, M. Mirza, Z. Wang, K. Xu, and J. He, Nano Lett. 13, 5344 (2013).

[25] A. A. Taskin, F. Yang, S. Sasaki, K. Segawa, and Y. Ando, Phys. Rev. B 89, 121302(R) (2014).

[26] S. Mizukami, K. Ando, and T. Miyazaki, J. Magn. Magn. Mater. 239, 42 (2002).

[27] Y. Tserkovnyak, A. Brataas, and G. E. W. Bauer, Phys. Rev. Lett. 88, 117601 (2002).

[28] K. Ando, S. Takahashi, J. Ieda, H. Kurebayashi, T. Trypiniotis, C. H. W. Barnes, S. Maekawa, and E. Saitoh, Nat. Mater. 10, 655 (2011).

[29] L. Chen, F. Matsukura, and H. Ohno, Nat. Commun. 4, 2055 (2013).

[30] J. Lustikova, Y. Shiomi, and E. Saitoh, Phys. Rev. B 92, 224436 (2015).

[31] S. M. Rezende, R. L. Rodríguez-Suárez, R. O. Cunha, A. R. Rodrigues, F. L. A. Machado, G. A. Fonseca Guerra, J. C. Lopez Ortiz, and A. Azevedo, Phys. Rev. B 89, 014416 (2014).

[32] S. Wang, L. Zou, X. Zhang, J. Cai, S. Wang, B. Shen, and J. Sun, Nanoscale 7, 17812 (2015).

[33] R. Ishikawa, T. Yamaguchi, Y. Ohtaki, R. Akiyama, and S. Kuroda, J. Cryst. Growth 453, 124 (2016).

[34] E. C. da Silva, R. Meckenstock, O. von Geisau, R. Kordecki, J. Pelzl, J. A. Wolf, and P. Grünberg, J. Magn. Magn. Mater. 121, 528 (1993).

[35] T. Toliński, K. Lenz, J. Lindner, E. Kosubek, K. Baberschke, D. Spoddig, and R. Meckenstock, Solid State Commun. 128, 385 (2003).

[36] See Supplemental Material at http://link.aps.org/supplemental/ 10.1103/PhysRevB.96.094424 for calculation of the ferromagnetic resonance conditions (Sec. I), shape of the FMR absorption spectra (Sec. II), and behavior of the antisymmetric Lorentzian components of the electromotive force (Sec. III).

[37] A. Azevedo, L. H. Vilela Leão, R. L. Rodriguez-Suarez, A. B. Oliveira, and S. M. Rezende, J. Appl. Phys. 97, 10 C715 (2005).

[38] E. Saito, M. Ueda, H. Miyajima, and G. Tatara, Appl. Phys. Lett. 88, 182509 (2006).

[39] S. Dushenko, H. Ago, K. Kawahara, T. Tsuda, S. Kuwabata, T. Takenobu, T. Shinjo, Y. Ando, and M. Shiraishi, Phys. Rev. Lett. 116, 166102 (2016)

[40] For example, M. L. Yu and J. T. H. Chang, J. Phys. Chem. Solids 31, 1997 (1970).

[41] Y. Shiomi, K. Nomura, Y. Kajiwara, K. Eto, M. Novak, K. Segawa, Y. Ando, and E. Saitoh, Phys. Rev. Lett. 113, 196601 (2014).

[42] D. H. Damon, J. Appl. Phys. 37, 3181 (1966).

[43] K. Ando and E. Saito, J. Appl. Phys. 108, 113925 (2010).

[44] P. Deorani, J. Son, K. Banerjee, N. Koirala, M. Brahlek, S. Oh, and H. Yang, Phys. Rev. B 90, 094403 (2014). 